\title{
Correspondance
}

\section{Mesothelioma and venous thrombosis}

A mi Schattner and Natasha Kozack describe and illustrate a unique and unfamiliar physical sign. ${ }^{1}$ Better-known differential diagnoses for enlarged supraclavicular fossae include obesity and Cushing's syndrome. The one time that I observed such enlargement unilaterally was in an obese woman who had lost much supraclavicular soft-tissue volume through remote full radical mastectomy. 'This patient's cortisol levels were normal.

With regard to Virchow's triad, the injury that predisposes to thrombosis damages the endothelium, not the epithelium, and the specific abnormality of blood flow is stasis. In the case described by Schattner and Kozack, ${ }^{1}$ lack of flow was likely fostered not merely by proximity of the tumour but also by extrinsic compression of the internal jugular vein or any of the more proximal veins, such as the left innominate vein. The resultant venous stasis and venous hypertension would be transmitted to the internal jugular vein, which would in turn predispose to thrombosis of the latter. Alternatively, primary thrombosis that occurred proximally might have been propagated distally.

It would be of interest if Schattner and Kozack could report the state of the great veins at the time of surgery, on the basis of either imaging or direct observation during the operation, as well as the local findings if an autopsy was performed when the patient died, 3 months later.

\section{Henry Schneiderman}

Hebrew Health Care

University of Connecticut School of Medicine

West Hartford, Conn.

\section{References}

1. Schattner A, Kozack N. A 47-year-old man with mesothelioma and neck swelling. CMAJ 2004; 170(4):465.

2. Schneiderman H. Severely disfiguring therapy for breast cancer, and the evolution of medicine. Consultant 1997;37:1006-14.

DOI:10.1503/cmaj.1040364

A mi Schattner and Natasha Kozack report a case of mesothelioma in a man who exhibited jugular and subclavian vein thrombosis. ${ }^{1}$ Malignant pleural mesothelioma is a relatively rare type of cancer that is reported to be associated with venous thromboembolism. ${ }^{2}$ We describe here a patient with malignant pleural and peritoneal mesothelioma who experienced portal vein thrombosis. This patient had a history of exposure to zeolite, and ascites developed while the patient was being followed for the malignant pleural mesothelioma.

CT of the abdomen revealed diaphragmatic invasion of the right pleural mesothelioma. In addition to the peritoneum, the omentum and mesentery were diffusely invaded by the tumour. At the same level, the portal vein and the inferior vena cava both appeared to have a lucent lumen and exhibited contrast enhancement at the vessel wall (Fig. 1).

Cancer patients are prone to thromboembolic events, either as part of a paraneoplastic syndrome or because of a decrease in anticoagulation factors, such as prothrombin $\mathrm{C}$, prothrombin $\mathrm{S}$ and antithrombin III, caused by some chemotherapeutic agents. ${ }^{3}$ Mesothelioma cells and cell lines have been shown to secrete a pleiotropic cytokine,

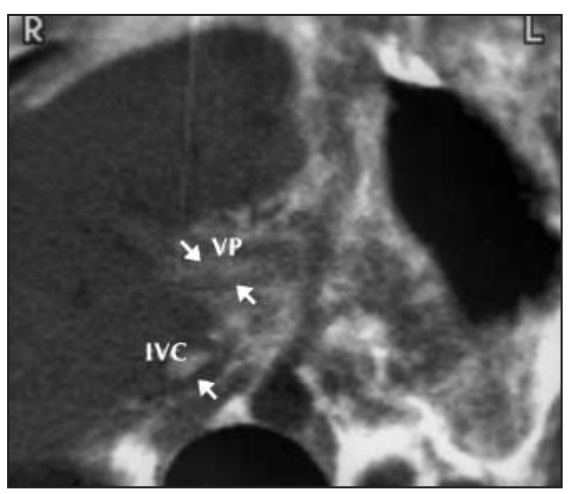

Fig. 1: Transverse CT scan demonstrates thrombosis (arrows) of both the portal vein (VP) and the inferior vena cava (IVC). interleukin 6 , which potentiates the production of platelets. ${ }^{4}$ In addition, mesothelioma cells release a sedimentable procoagulant material in vitro; ${ }^{5}$ however, the underlying mechanism of thrombosis in mesothelioma is still a mystery.

Physicians who care for patients with mesothelioma should keep in mind that the thrombotic complication of the disease is not rare and may also involve a relatively unusual site, the portal system.

\section{Mehlika Isildak}

Gulay Sain Guven

Department of Internal Medicine

\section{Salih Emri}

Department of Respiratory Medicine

Hacettepe University Faculty

of Medicine

Ankara, Turkey

\section{References}

1. Schattner A, Kozack N. A 47-year-old man with mesothelioma and neck swelling. CMAf 2004; 170(4):465.

2. Chahinian AP, Pajak TF, Holland JF, Norton L Ambinder RM, Mandel EM. Diffuse malignant mesothelioma. Prospective evaluation of $69 \mathrm{pa}-$ tients. Ann Intern Med 1982;96:746-55.

3. Rogers JS 2nd, Murgo AJ, Fontana JA, Raich JC. Chemotherapy for breast cancer decreases plasma protein C and protein S. 7 Clin Oncol 1988;6:276-81.

4. Nakano T, Chahinian AP, Shinjo M, Tonomura A, Miyake M, Togawa N, et al. Interleukin 6 and its relationship to clinical parameters in patients with malignant pleural mesothelioma. $\mathrm{Br} 7$ Cancer 1998;77:907-12.

5. Idell S, Pueblitz S, Emri S, Gungen Y, Gray L, Kumar A, et al. Regulation of fibrin deposition by malignant mesothelioma. Am 7 Pathol 1995; 147:1318-29.

DOI:10.1503/cmaj.1040664

\section{[One of the authors responds:]}

T $T$ e agree with Henry Schneiderman that in the patient we described $^{1}$ the proximity of the tumour to the thrombosed vessels was far from coincidental and that the tumour was likely material to the pathogenesis of thrombosis. One of 2 mechanisms could be involved: either, as Schneiderman suggests, mechanical compression of proximal veins causing venous stasis or elaboration by the tumour cells of spe- 